\title{
ORIGINAL RESEARCH \\ Carotid Body Tumors: Objective Criteria to Predict the Shamblin Group on MR Imaging
}

S. Arya

V. Rao

S. Juvekar

A.K. Dcruz
BACKGROUND AND PURPOSE: MR imaging is an established method for diagnosis and surgical planning of carotid body tumors (CBTs). However no studies have elaborated preoperative objective criteria to predict the Shamblin (surgical) classification of CBTs, an important predictor of vascular morbidity. The purpose of this study was to establish criteria to accurately predict the Shamblin group on preoperative MR imaging for a uniform reporting system.

MATERIALS AND METHODS: MR images of 9 CBTs in 8 consecutive patients who underwent surgery between 2004 and 2007 were reviewed at a tertiary cancer hospital. The surgical records were blinded to the radiologists. A radiologic classification into 3 types (I, II, and III) based on the maximum degree of circumferential contact of the tumor with the internal carotid artery (ICA) was attempted and correlated with the Shamblin group in surgical records.

RESULTS: There were 5 type III, 3 type II, and 1 type I tumors. The type I tumor had an ICA maximum circumference of contact less than or equal to $180^{\circ}$, type II tumors had more than $180^{\circ}$ and less than $270^{\circ}$, and type III tumors had a maximum circumference of contact of $270^{\circ}$ of more. MR imaging accurately predicted the Shamblin group in $8(100 \%)$ of 8 operated tumors. Tumor size and Shamblin group did not have a uniformly predictable relation.

CONCLUSIONS: Shamblin group can be predicted preoperatively on MR imaging, and the maximum degree of circumferential contact of the CBT with the ICA on axial images is the criterion to predict the Shamblin group.
C rotid body tumors (CBTs) account for more than $50 \%$ of head and neck paragangliomas. ${ }^{1}$ The preferred method of treatment is surgery, which can be challenging because the tumor is a highly vascular mass that is often densely adherent to the carotid bifurcation. Meticulous preoperative planning and careful patient selection are essential for a successful surgical outcome. A landmark in the assessment of resectability of these tumors is the surgical classification by Shamblin et $\mathrm{al}^{2}{ }^{2}$ which continues to be a predictor of vascular morbidity. Shamblin et $\mathrm{al}^{2}$ classified these tumors into 3 groups based on the operative notes and gross specimen examination and established that the risk of surgical intervention depends mainly on the relationship of the tumor with the carotid vessels (Fig 1). The importance of this classification increases significantly if preoperative (noninvasive) cross-sectional imaging can accurately predict the Shamblin group. MR imaging is a frequently used imaging method in the diagnosis and preoperative work-up of these tumors and provides relevant information for surgical planning. ${ }^{3}$ However, most reports on the diagnosis and surgical management of CBTs do not mention specific or consistent imaging criteria to predict this classification. Some authors have also stated that CBTs cannot be classified preoperatively. ${ }^{4}$ van der Mey et $\mathrm{al}^{5}$ have stressed the need for a uniform classification system for these tumors so that the communication in the literature could be consistent. The purpose of this study was to establish objective criteria to

Received November 14, 2007; accepted after revision February 6, 2008.

From the Departments of Radiology (S.A., S.J.) and Head and Neck Surgical Oncology (V.R., A.K.D.), Tata Memorial Hospital, Mumbai, India

Please address correspondence to Supreeta Arya, Department of Radiology, Tata Memorial Hospital, Ernest Borges Rd, Parel, Mumbai 400 012, India; e-mail: supreeta.arya@gmail.com

$\equiv$ E indicates article with supplemental on-line table.

DOI 10.3174/ajnr.A1092 predict the Shamblin classification after reviewing the imaging records.

\section{Materials and Methods}

\section{Patients}

A retrospective review of the MR images of 9 CBTs in 8 patients between 2004 and 2007 was performed. The chosen period of 20042007 coincided with the installation of the PACS in 2004 at our center. The age range of the 8 patients was $27.0-57.0$ years, with a mean of 35.5 years. Five were male and 3 were female. One patient had bilateral tumors. None of the cases were familial tumors. Surgery had been attempted in 8 tumors. The left-sided tumor in the patient with bilateral tumors was not operated on. Histopathologic examination after surgical resection confirmed the diagnosis of CBTs in all of the cases.

\section{MR Imaging}

MR imaging in all of the cases had been performed on a $1.5 \mathrm{~T}$ magnet. The MR images were reviewed on the PACS. The MR images acquired were axial and coronal spin-echo T1-weighted (TR, 330-600 ms; TE, 8-15 ms); axial and sagittal fast spin-echo T2-weighted (TR, 3500$4500 \mathrm{~ms}$; TE, 80-110 ms); coronal and sagittal short tau inversion recovery (STIR) (inversion time, $150 \mathrm{~ms}$ ); and postcontrast axial, coronal, and sagittal T1-weighted sequences. A dedicated neck surface coil was used. The sequences were acquired at 4-mm thickness in all of the cases with no intersection gap. Postcontrast studies had been performed after injection of $0.1 \mathrm{mmol} / \mathrm{kg}$ of gadolinium-diethylenetriaminepentaacetic acid. The matrix used was $256 \times 256$, and the FOV was $220 \mathrm{~mm}$. MR angiography had been performed in all of the cases by using either $2 \mathrm{D}$ time-of flight (TOF) or 3D TOF sequences (TR, 25-35 ms; TE, 4-8 ms), with a section thickness of $1 \mathrm{~mm}$.

\section{Image Analysis}

The information recorded included tumor location, splaying of the carotid bifurcation, characteristic MR imaging features, axial dimensions, 

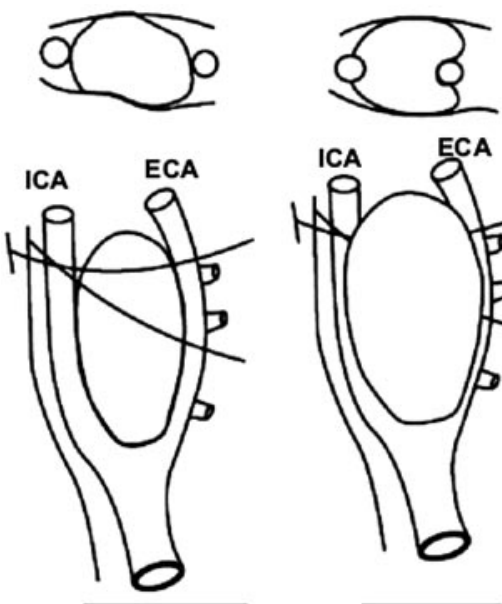

Type I

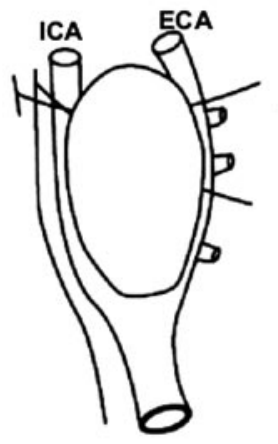

Type II
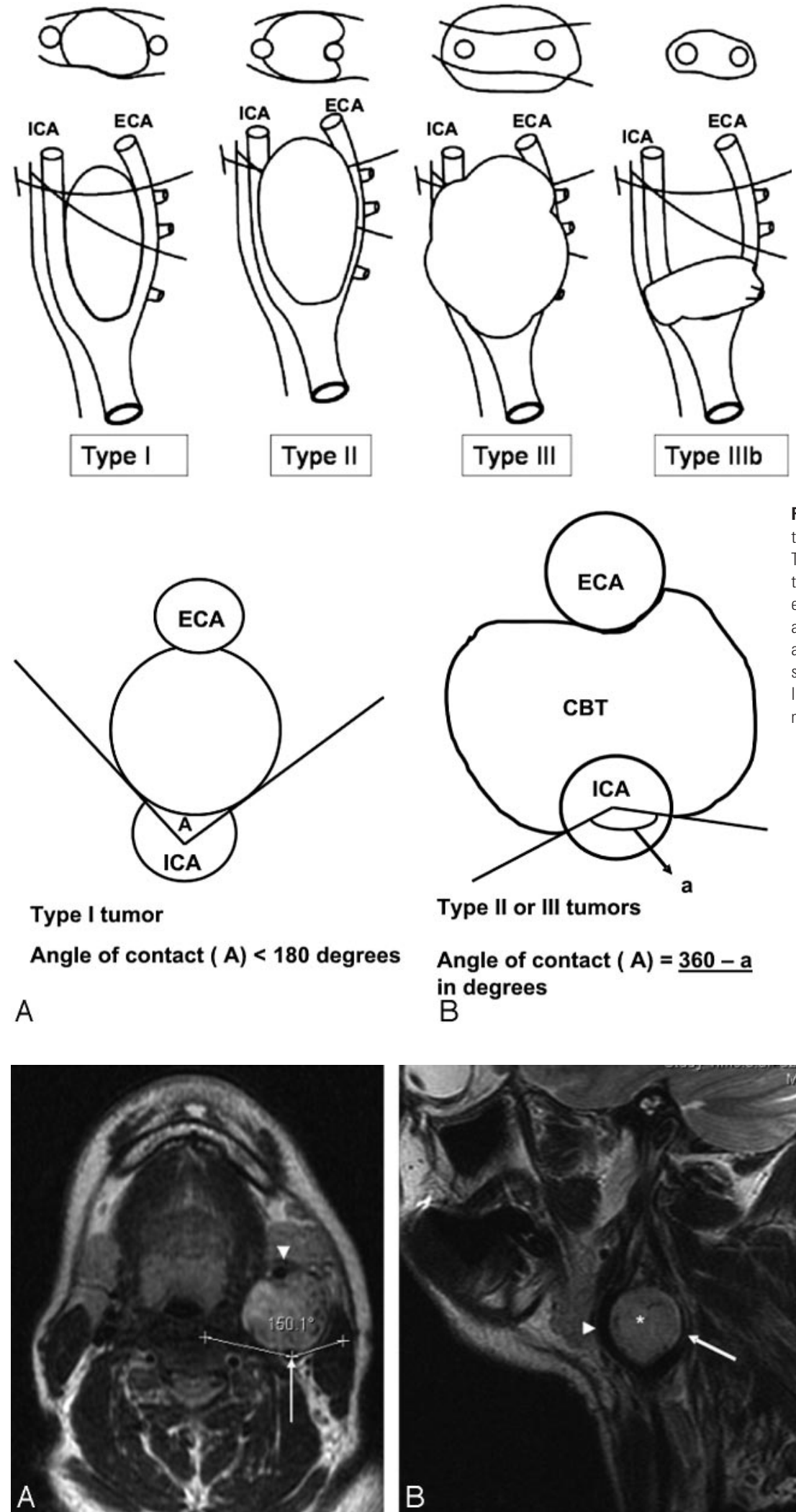

Type II or III tumors

Type I tumor

Angle of contact $(A)<180$ degrees

A
Angle of contact $(A)=\underline{360-a}$ in degrees

B

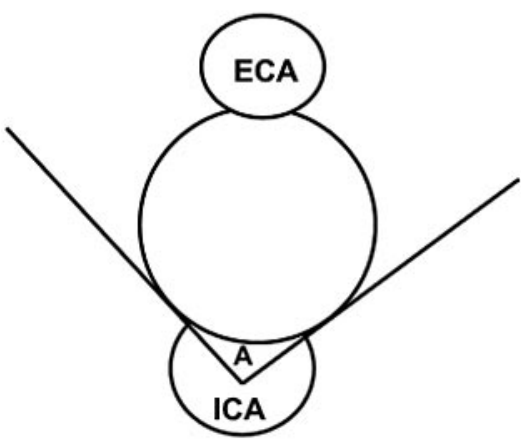

A

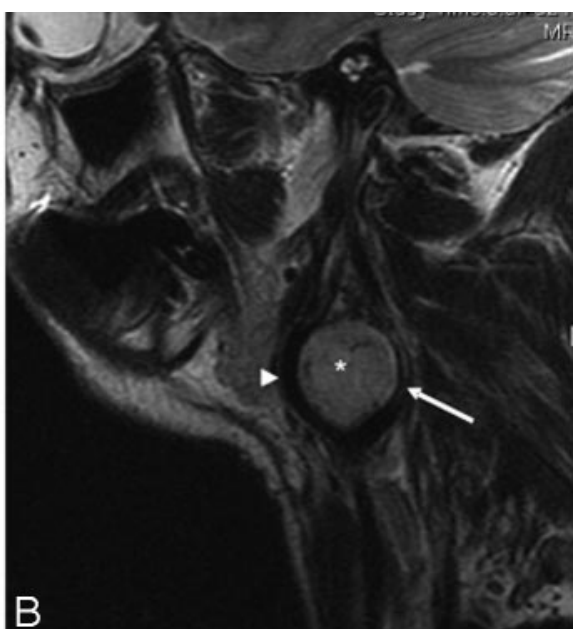

Fig 1. Shows a schematic diagram of the Shamblin grouping of CBTs into I, II, and III, as well as IIIb (as proposed in the modification to Shamblin's classification by Luna-Ortiz et al ${ }^{4}$. The surgical grouping is chiefly based on the relationship of the tumor to the carotid vessels, ICA and ECA. The class IIIb tumors include tumors of any size that are intimately adherent to the carotid vessels. The oblique lines shown represent the $\mathrm{X}$ and $\mathrm{XII}$ nerves, which are intimately related to the tumors and have to be carefully dissected along with the vessels.
Fig 2. A schematic diagram demonstrating the measurement of the degree of circumferential contact between ICA and the tumor. Two intersecting lines are drawn between the center of ICA and the points of contact of the circumference of the vessel with the edges of the tumor to obtain the angle $(A)$. $A$, Type I tumor: the angle " $\mathrm{A}$ " is depicted between the 2 intersecting lines. $B$, Type I and III tumors: the angle measurement tool automatically measures the smaller angle (less than $180^{\circ}$ ) between the intersecting lines. The actual angle $\mathrm{A}$ is the difference between $360^{\circ}$ and measured angle "a" $\left(360^{\circ}-a\right)$, as shown here. and the craniocaudal extent of the tumor from which the tumor volume was calculated, the distance of the superior limit of tumor from the skull base, and specifically the degree of circumferential contact and length of contact of the tumor with internal, external, and common carotid arteries. The degree of circumference of contact was measured with the inbuilt angle measurement tool on the PACS system on the axial images. The maximum degree of contact was recorded in each case. We used both axial T2-weighted and postcontrast T1-weighted sequences for the measurement and found the measurements comparable on both sequences. The circumference of contact was calculated by measuring the angle between 2 intersecting lines passing from the center of the vessel to the circumference of the vessel in contact with the tumor (Fig 2). Because the 


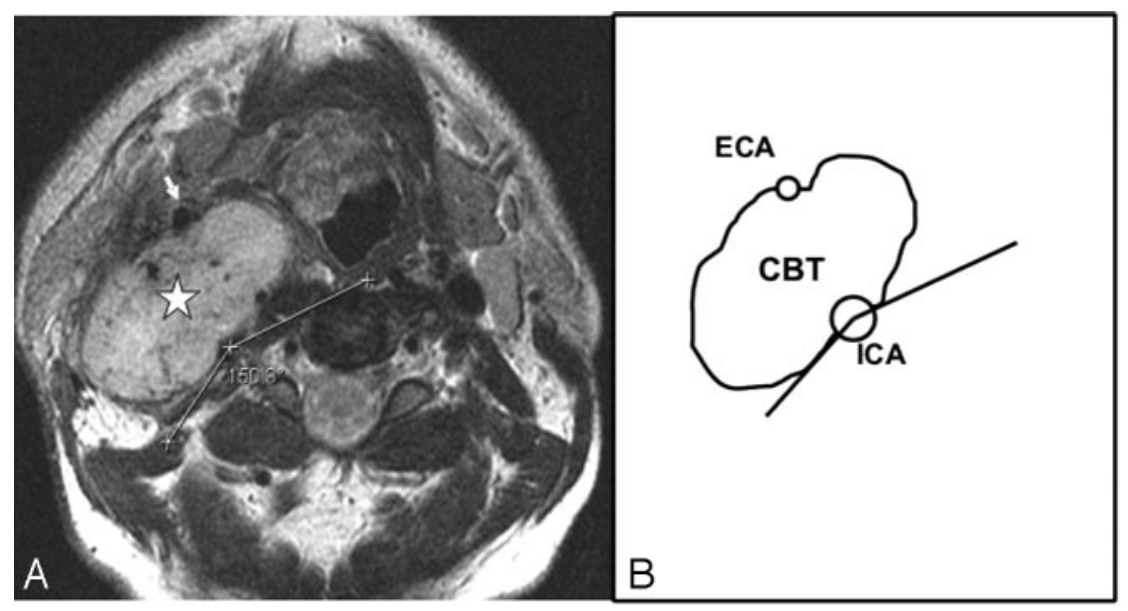

Fig 4. Type II CBT. A, Axial T2-weighted MR image showing a type II right-sided carotid body tumor (star) splaying the ICA posteriorly and ECA (arrow) anteriorly. The circumferential degree of contact of the tumor with the ICA is the difference between $360^{\circ}$ and the measured angle $\left(150^{\circ}\right)$ in the figure, that is, $210^{\circ}$. B, Line diagram illustrating the tumor-ICA circumference of contact of the type II CBT in $A$.

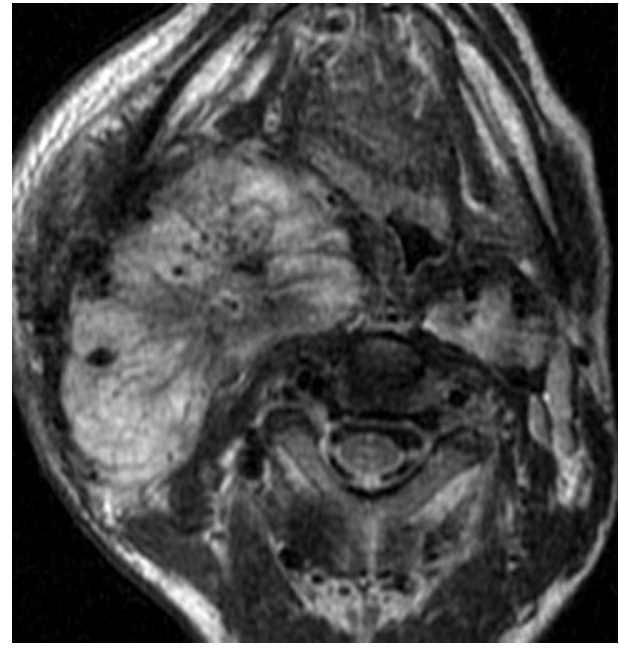

Fig 5. A bulky type III CBT. Axial T2-weighted MR image showing a type III right-sided CBT as a brightly hyperintense mass with multiple flow voids. The ICA and ECA are both completely encased $\left(360^{\circ}\right.$ circumference of contact).

relation of the tumor to the internal carotid artery (ICA) is most critical for surgical planning, we used the circumferential degree of contact of the tumor with the ICA (A1) to classify the tumor radiologically into 3 types in an attempt to predict the Shamblin group of the tumors: type I, A1 less than or equal to $180^{\circ}$; type II, Al greater than $180^{\circ}$ and less than $270^{\circ}$; and type III, Al greater than or equal to $270^{\circ}$. The degree of circumference of contact of the tumor with the external carotid artery (ECA; A2) and, where applicable, with the common carotid artery (CCA; A3) and the tumor size/volume was not incorporated in this classification.

With type I tumors, A1 was measured directly (Figs $2 A$ and 3 ), whereas with the type II and III tumors, A1 was obtained by deducting the measured angle (degree of circumference not in contact) from $360^{\circ}$ (Figs $2 B$ and 4 ). This was done because when A1 exceeded $180^{\circ}$, the angle measurement tool automatically measured the smaller angle formed between the 2 intersecting lines (Fig $2 B$ ). If the tumor encased the entire vessel, A1 was recorded as $360^{\circ}$ (Figs 5 and 6).

Volume estimation was performed with the formula for an ellipse (length $\times$ breadth $\times$ thickness $\times 0.5$ ), because all of the tumors were elliptical. We used volume rather than a unidimensional measurement, because it most accurately reflects the size in these elliptical tumors.

\section{Comparison with Surgical Records}

The surgical records were blinded to the radiologists while they reviewed the MR images. The information obtained on the MR imaging

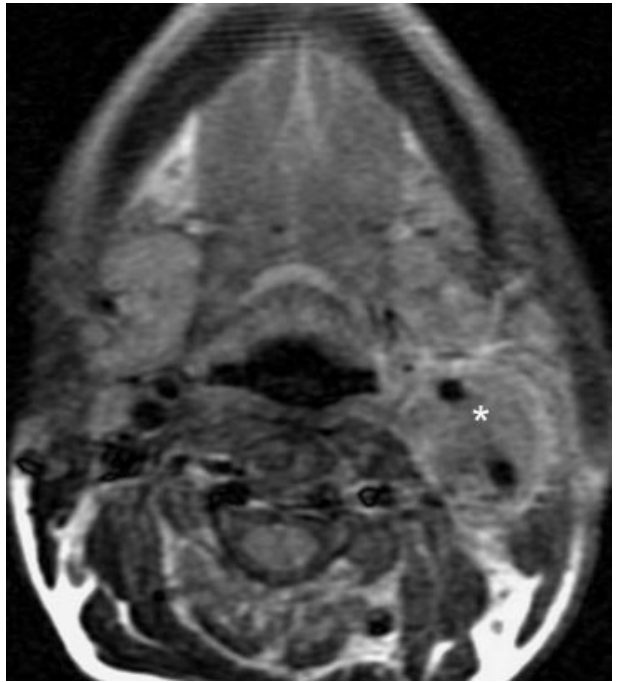

Fig 6. Small-volume type III CBT. Axial T2-weighted MR image showing a left-sided CBT $\left({ }^{*}\right)$ with complete encasement of ICA and ECA

review was compared with the surgical record in each case, which included the Shamblin group of the tumor. The Shamblin group of the tumor was classified intraoperatively based on at least 2 of the following criteria: 1) the extent of circumferential encasement of the carotid vessels by the tumor as seen intraoperatively; 2) the feasibility of obtaining a plane of dissection between tumor and the vessel; and 3 ) the presence of adventitial infiltration on gross examination. A Shamblin I tumor encased the vessels partially and could be easily dissected from the vessels, which did not show adventitial infiltration. Shamblin III tumors encased the vessels almost completely, and despite prolonged and meticulous dissection, inability to find a plane between the tumor and vessels necessitated ICA resection. There was obvious adventitial infiltration. Shamblin II tumors were difficult to dissect from the vessels, which had partial or focal adventitial infiltration. However, it was possible to preserve the ICA despite minimal damage to the wall in some cases.

\section{Results}

\section{MR Imaging Review}

There were 9 CBTs in 8 patients, with bilateral tumors in 1 patient. T2-weighted fat-suppressed sequences depicted all of the tumors as hyperintense elliptical masses splaying the CCA bifurcation and with punctuate flow voids. Seven of the tumors splayed the arteries in an anteroposterior direction, 
whereas 2 tumors splayed the vessels medially and laterally. Postcontrast fat-suppressed T1-weighted images showed intense enhancement in all of the tumors. The location at the carotid bifurcation with the splaying of arteries and the intense enhancement were the diagnostic features. None of the tumors displayed intracranial extension. None of the patients had any evidence of nodal metastases.

Based solely on the degree of circumferential contact of the tumor with the ICA (A1), we classified 5 CBTs as type III, 3 as type II, and 1 as type I (On-line Table 1). Because tumor size and relation with vessels are both components of Shamblin classification, correlation between the radiologic type and tumor volume was attempted. Tumor volume in the 9 CBTs ranged from 14 to $180 \mathrm{~mL}$. Of the 5 tumors classified as type III, 3 had a volume equal to or less than $20 \mathrm{~mL}$, the smallest with a volume of $14 \mathrm{~mL}$. However, 2 of the smallest tumors were seen completely encasing the ICA (Fig 6). The largest type III tumor was bulky, with a volume of approximately $180 \mathrm{~mL}$, and completely encased the distal ICA and the entire ECA ( $\mathrm{A} 1$ and $\mathrm{A} 2=360^{\circ}$ ). The tumor spilled below the bifurcation along the CCA for a length of $3 \mathrm{~cm}$ with a $190^{\circ}$ angle of contact. Three tumors labeled type II on MR imaging (each with $\mathrm{A} 1$ of $210^{\circ}$ ) had a volume ranging between 22.5 and $26.8 \mathrm{~mL}$. The solitary type I CBT had a volume of 15.8 $\mathrm{mL}$. The distance between the superior limit of the tumor and the skull base, which indicates the available free segment of ICA needed for anastomosis of the graft, varied from 1.6 to $5.5 \mathrm{~cm}$ (On-line Table 1).

\section{Comparison between Surgical Records and MR Imaging Review}

Type III Tumors. Surgical records showed that in 4 of 5 of the type III tumors, complete resection of the tumor was achieved. However, the tumor was intimately adherent to the ICA in all 4 necessitating ICA resections, and continuity was reestablished with graft. The ECA was ligated in 3 of the 4 cases, and MR imaging records revealed a circumference of contact (A2) more than or equal to $270^{\circ}$. In the fourth case, where the ECA was preserved, A2 was $180^{\circ}$. In the fifth bulky type III tumor (volume, $180 \mathrm{~mL}$ ), surgical resection was incomplete. The upper part of the tumor completely encased both ECA and ICA, reaching close to the skull base. To achieve complete resection, the ICA needed to be resected and reanastomosed; however, the available free segment of distal ICA was inadequate for such anastomosis. However, the distal part of the tumor abutting the CCA could be dissected from the vessel and was resected. The CCA was preserved. The duration of the surgery was prolonged, and massive blood transfusion was required.

Type II Tumors. One of the type II tumors was not operated on. The other 2 tumors were completely resected, with preservation of ICA in both. However, in 1 patient, the tumor could not be focally dissected off the ICA wall near the bifurcation, and a part of the vessel wall was removed with repair of the rent with prolene. In the other patient, the tumor was intimately adherent to both carotid vessels at places and was dissected off the ICA with difficulty. However, the external carotid artery was ligated $\left(\mathrm{A} 2=260^{\circ}\right)$.

Type I Tumor. Surgical records showed that the solitary type I tumor could be easily separated from the ICA, ECA, and the vagus nerve, all of which were preserved, and complete resection of the tumor was achieved. A2 was $180^{\circ}$, whereas A1 was $150^{\circ}$.

Free Distal Segment of ICA. In the type III CBT, where resection was incomplete, MR imaging accurately measured the inadequate free distal segment of ICA $(1.6 \mathrm{~cm})$. Resection was incomplete, because the surgical decision was against ICA sacrifice.

Complete surgical resection of the CBT had been achieved in 7 of the 8 operated cases. MR imaging successfully predicted the Shamblin group (and vascular outcome) in 8 (100\%) of 8 operated cases. In the 7 patients with curative resection, a total of 8 vessels (4 ICAs and 4 ECAs) were resected/ligated. In all except 1 , the $\mathrm{A} 1$ and $\mathrm{A} 2$ values were $270^{\circ}$ or more. In the last, the A2 value was $260^{\circ}$.

\section{Discussion}

Surgery, which is the curative method of treatment for CBTs, requires complete excision of the tumor, with preservation of vital neurovascular structures. ${ }^{3,6,7}$ Radiation therapy is a treatment option only for CBTs that are advanced and unresectable, as well as in multiple tumors. ${ }^{7-9}$ The main factors influencing surgical treatment of CBTs, apart from age and operative risk, are multifocality and the possibility of impairment of cranial nerves and injury to ICA. ${ }^{3,7}$ Shamblin et al, ${ }^{2}$ in the 1970s suggested a surgical classification of CBTs into 3 groups. This classification was according to the gross tumorvessel relationship and was based on the intraoperative findings and postoperative specimen examination. Group 1 tumors were minimally attached to the vessels and easily resectable. Group 2 tumors seemed to partially surround the vessel and were more adherent to vessel adventitia. These tumors were difficult to dissect but amenable to careful resection. Shamblin group 3 tumors had an intimate adherent relationship to the entire circumference of the carotid bifurcation, and surgical dissection was impossible even in the hands of experienced vascular surgeons. This group, therefore, required sacrifice of the ICA with vessel replacement. This classification has been widely used by subsequent authors as a predictor of vascular morbidity and for surgical decisionmaking. ${ }^{3-5}$ A directly proportional relationship of the Shamblin group with blood loss and surgical time has also been reported.

Attempts have been made to preoperatively classify the CBTs with imaging mainly with arteriography. The identification of a clear lucency between the tumor and the ICA indicates the probability of a Shamblin group 1 tumor. $^{3}$ If the tumor completely encases the bifurcation without any visible lucency, the tumor is probably a group 3 lesion. Others have attempted to predict the kind of surgery preoperatively by measuring the carotid bifurcation angle or degree of splaying of the carotid arteries on arteriography. An angle less than $90^{\circ}$ implied that the tumor can be dissected off the ICA, whereas more than $90^{\circ}$ indicates the probability of en bloc resection and carotid reconstruction. ${ }^{10}$ However, since its advent, MR imaging is widely used in evaluating CBTs. ${ }^{11-13}$ The classic MR imaging findings are a hyperintense mass on T2-weighted sequences with flow voids splaying the carotid bifurcation. Postcontrast sequences show striking early phase arterial enhancement. MR angiography is particularly useful in diagnosis when 
the tumors are multiple, as in familial cases. ${ }^{14}$ Although MR imaging has proven itself valuable as a noninvasive technique for diagnosis ${ }^{11-13}$ and may indicate the Shamblin group, this is sometimes a subjective diagnosis, and no objective criteria that can be uniformly applied have been described in the literature. A Shamblin group 3 tumor that encases vessels completely may be obvious on MR imaging. Some prefer to assess the intracranial circulation for collaterals when ICA encasement by the CBT exceeds $270^{\circ},{ }^{5}$ suggesting that this implies a group 3 tumor, which may require carotid sacrifice, but imaging criteria for group 1 and 2 are not clear.

A recent report also mentions that, although attempts have been made to classify them, CBTs cannot be classified preoperatively, because tumor infiltration to the carotid wall can be perceived only during surgery. ${ }^{4}$ However, axial cross-sectional imaging can clearly demonstrate the circumferential involvement of the tumor with the vessels, and so we hypothesized that by measuring the degree of circumference of contact of the tumor with the ICA, we could probably predict the Shamblin group. We used the tumor-ICA relation alone in our classification and analysis because this is the most important vessel requiring preservation in curative resection. Previous authors have studied the resectability of advanced head and neck squamous cancers with respect to the carotid vessels and have identified circumferential contact greater than $270^{\circ}$ between tumor and vessel to indicate an inability to strip off the primary/nodal disease from the CCA adventitia. ${ }^{15}$ Others have indicated that, with a degree of circumferential contact between tumor and vessel less than $180^{\circ}$, the tumor can be peeled off the vessel. ${ }^{16}$ Although CBTs and squamous carcinomas are entirely different entities, we extrapolated these criteria for radiologically classifying the CBTs in an attempt to aid preoperative decision-making. Although the degree of vessel wall infiltration would be perceived only at surgery or on histology, ${ }^{4}$ the above-mentioned studies in squamous cancers of the head and neck led us to hypothesize that an increasing degree of circumference of contact between tumor and vessel could probably indicate a greater degree of wall infiltration.

In our series, the attempted radiologic classification corresponding with the Shamblin group correlated accurately with the intraoperative findings and vascular outcome. Our study clearly demonstrates that, with progressive increase in the circumference of contact of the ICA/ECA with the tumor beyond $180^{\circ}$, as seen on MR imaging, the ease of resection diminishes, and the likelihood of vascular damage increases, with a need for vessel resection particularly arising when the angle exceeds $270^{\circ}$. This was true for tumors of any size. Although it is known that surgery is difficult with increasing tumor volumes and is likely to result in neurovascular injury, ${ }^{2,3,5,7}$ our findings indicate that the relationship of the tumor with the walls of the ICA is more important. A feature to note is that, whereas many surgical reports ${ }^{2,4}$ mention circumferential involvement of the carotid bifurcation (ICA and ECA) to be group 3, involvement of three fourths and more of the circumference of the ICA on axial MR images necessitates vessel resection, and a tumor with $\mathrm{A} 1$ of $270^{\circ}$ or more would qualify as group 3 regardless of the $\mathrm{A} 2$ value.

Although the original report by Shamblin et $\mathrm{al}^{2}$ did not specify the exact size of the tumors, it did mention a linear relationship between the tumor size and Shamblin group.
However, Shamblin also clearly states that the tumor-vessel wall relationship in their series of 58 resections was variable and not always related to size. Subsequent authors have specified a cutoff size of $4 \mathrm{~cm}$ to classify between "type" I (instead of group 1) Shamblin tumors $(<4 \mathrm{~cm})$ and Shamblin II and III types. ${ }^{17}$ CBTs are usually elliptical, but these reports do not clarify the axis used when specifying $4 \mathrm{~cm}$. With cross-sectional imaging, an inherent advantage is the preoperative availability of measurements in all 3 of the axes and the possibility of volume estimation. More information regarding the volume of CBTs is needed in future imaging literature to specify any cutoff volumes. However, our series did not reveal a uniformly predictable relation between the radiologic type (which correlated accurately with the Shamblin type) and the tumor volume. Large tumors are more likely to be Shamblin type II or III, but small tumors need not be Shamblin type I. We found the smallest tumor in our series to infiltrate the ICA circumferentially. Luna-Ortiz et $\mathrm{al}^{4}$ have made a similar observation and found $22 \%$ of tumors in their series of 50 CBTs to be small tumors but infiltrating the carotid vessels to an extent requiring ICA sacrifice and reanastomosis with graft. They proposed a modification of the Shamblin classification and suggest that a CBT of any size, if intimately adherent to the vessels, should be classified as modified Shamblin class IIIb, whereas IIIa represents the original III described by Shamblin. Shamblin's classification does not predict neurologic morbidity, whereas a correlation is known to exist between larger tumors, particularly $>4 \mathrm{~cm}$, and neurologic damage. ${ }^{4,5,17}$ The suggested modification, therefore, aims to predict both vascular and neurologic morbidity by incorporating both size and tumor-vessel relation.

We did not use tumor size in the MR imaging classification of CBTs. Retrospective analysis revealed that incorporating volume in the radiologic classification to predict the Shamblin group would have been misleading in our attempt to predict the vascular outcome with respect to the ICA (which is the most important component of Shamblin classification). In our series, some of the smallest tumors belonged to Shamblin type III, whereas 1 of the tumors labeled type II in our series with a maximum axial dimension of $6.3 \mathrm{~cm}$ (volume, $26.8 \mathrm{~mL}$ ) was resected completely with preservation of the ICA $\left(\mathrm{A} 1,210^{\circ}\right)$. These observations highlight the nonlinear relation between size and Shamblin type, particularly in small- and medium-sized tumors. However as tumor size increases even further, there is the likelihood of greater vessel encasement, and the relationship becomes directly proportional. The possible cause for a greater circumference of contact of the tumor with the ICA and/or ECA, even in small-volume tumors, may lie in the position of the CBT. Both adventitial and periadventitial locations for the carotid body (from which the tumor arises) have been described. ${ }^{1,18}$ It may either be located along the ICA or even along the ECA. A tumor arising from a carotid body located within the adventitia is more likely to develop a larger degree of circumference of contact with the vessel and possibly earlier when the tumor is small. However this hypothesis needs histopathologic correlation in future prospective series.

Variations in practice exist between institutions regarding treatment of CBTs, especially with respect to radical 
surgery requiring carotid sacrifice and replacement with graft. Although radical surgery may be attempted at some centers, others seldom practice carotid sacrifice, and alternative forms of treatment may be offered, including radiation therapy and stereotactic radiosurgery. ${ }^{1,3,6-8,11}$ The problem with complete surgical resection of Shamblin type 3 tumors is the danger of sacrificing the ICA, of which patency, if not restored, has a high mortality and morbidity. Even after a successful balloon occlusion test, stroke rates have still been of the order of $25 \% .{ }^{5}$ Therefore, the feasibility of vascular reconstruction is another important factor influencing the surgical management of CBTs. A relevant important feature that needs to be recorded on the MR images is the distance between the superior limit of tumor and the skull base. This is important to identify the free distal stump of ICA needed for anastomosis of the graft if the continuity of the ICA is to be maintained. Previous authors have mentioned the minimum segment of the stump required to be approximately $2.0 \mathrm{~cm}$ for vascular anastomosis. ${ }^{19}$ Others have specified a segment length of $1.0 \mathrm{~cm} .{ }^{20}$ An unfavorable tumor factor of less than $2.0 \mathrm{~cm}$ of distal stump of carotid can make reconstruction of the vessel improbable despite a vascular team. ${ }^{19}$ Preoperative identification of this segment and its inadequacy could even tilt the decision in favor of nonsurgical therapy in a Shamblin type III tumor.

\section{Conclusion}

MR imaging is widely used for diagnosis and surgical planning of CBTs. However, despite a literature search, we did not come across any specific and consistent objective criteria to predict the Shamblin group on preoperative MR imaging, which is necessary for uniform consensus reporting. Our study is a review of a small number of these rare tumors, and, therefore, future prospective studies of a larger series would be worthwhile for statistical analyses before final conclusions can be made. In our small series, we found an excellent correlation between the radiologic prediction of the Shamblin group and the surgical outcome. The maximum degree of circumference of contact of the tumor with the ICA, as measured on axial MR images, should be the sole criterion to classify the Shamblin group, regardless of tumor size. On preoperative MR imaging, a tumor-ICA maximum degree of circumference of contact less than or equal to $180^{\circ}$ would represent Shamblin I, circumferential contact more than 180 and less than $270^{\circ}$ would represent Shamblin II, and greater than or equal to $270^{\circ}$ would represent Shamblin III. However, the size (or volume) is an independent factor requiring mention, because larger vol- umes are known to be associated with tedious surgery and neurologic injury. If the tumors are small and still display circumferential involvement of the ICA (IIIb of the proposed modification of Shamblin classification), this needs to be specifically conveyed to the surgeon, because the small size may be misinterpreted to signify greater ease of resection. The degree of circumferential contact with the ECA and, when applicable, the CCA predicts morbidity to these vessels. Lastly, measurement of the available distal free segment of ICA is vital information to plan the feasibility of vascular reconstruction if carotid sacrifice is inevitable.

\section{References}

1. Rao AB, Koeller KK, Adair CF. From the archives of the AFIP. Paragangliomas of the head and neck: radiologic-pathologic correlation Armed Forces Institute of Pathology Radiographics 1999;19:1605-32

2. Shamblin WR, ReMine WH, Sheps SG, et al. Carotid body tumor (chemodectoma). Clinicopathologic analysis of ninety cases. Am J Surg 1971;122:732-39

3. Knight TT Jr, Gonzalez JA, Rary JM, et al. Current concepts for the surgical management of carotid body tumor. Am J Surg 2006;191:104-10

4. Luna-Ortiz K, Rascon-Ortiz M, Villavicencio-Valencia V, et al. Does Shamblin's classification predict postoperative morbidity in carotid body tumors? A proposal to modify Shamblin's classification. Eur Arch Otorhinolaryngol 2006;263:171-75

5. van der Mey AGL, Jansen JC, van Baalen JM. Management of carotid body tumors. Otolaryngol Clin North Am 2001;34:907-24, vi

6. Luna-Ortiz K, Rascon-Ortiz M, Villavicencio-Valencia V, et al. Carotid body tumors: review of a 20-year experience. Oral Oncol 2005;41:56-61

7. Pellitteri PK, Rinaldo A, Myssiorek D, et al. Paragangliomas of the head and neck. Oral Oncol 2004;40:563-75

8. Boedeker CC, Ridder GJ, Neumann HP, et al. Diagnosis and management of cervical paragangliomas: the Freiburg experience. Laryngorhinootologie 2004;83:585-92

9. Gregory C, Kasper RE, Welling ARW, et al. A multidisciplinary approach to carotid paragangliomas. Vasc Endovasc Surg 2007;40;467-74

10. Pantanowitz D, Davidge-Pitts K, Demetriades D. The significance of the carotid bifurcation angle in carotid body tumours. S Afr Med J 1991;80:318-21

11. Van den Berg R. Imaging and management of head and neck paragangliomas. Eur Radiol 2005;15:1310-18

12. Vogl R, Bruning H, Schedel, et al. Paragangliomas of the jugular bulb and carotid body: MR imaging with short sequences and Gd-DTPA enhancement. AJR Am J Roentgenol 1989;153:583-87

13. Olsen WL, Dillon WP, Kelly WM, et al. MR imaging of paragangliomas. AJR Am J Roentgenol 1987;148:201-04

14. Van den Berg R, Schepers A, de Bruine FT, et al. The value of MR angiography techniques in the detection of head and neck paragangliomas. Eur J Radiol 2004;52:240-45

15. Yousem DM, Hatabu H, Hurst RW, et al. Carotid artery invasion by head and neck masses: prediction with MR imaging. Radiology 1995;195:715-20

16. Yoo GH, Hocwald E, Korkmaz H, et al. Assessment of carotid artery invasion in patients with head and neck cancer. Laryngoscope 2000;110:386-90

17. Davidge-Pitts KJ, Pantanowitz D. Carotid body tumors. Surg Annu 1984;16:203-27

18. Maxwell JG, Jones SW, Wilson E, et al. Carotid body tumor excisions: adverse outcomes of adding carotid endarterectomy. J Am Coll Surg 2004;198:36-41

19. Lore, JM. Resection of portion of common and internal carotid arteries involved by cancer. In: An Atlas of Head and Neck Surgery, 3rd ed. Philadelphia: Saunders; 1988:1074-81.

20. de Vries EJ, Sekhar LN, Horton JA, et al. Yonas H. A new method to predict safe resection of the internal carotid artery. Laryngoscope 1990;100:85-88 